\title{
Different Approach of VIDEO Compression Technique: A Study
}

\author{
S. S. Razali \\ Faculty of Electronic and \\ Computer Engineering \\ Universiti Teknikal Malaysia \\ Melaka (UTeM) \\ K. A. A. Aziz \\ Faculty of Engineering \\ Technology \\ Universiti Teknikal Malaysia \\ Melaka (UTeM)
}

\author{
N. M. Z. Hashim \\ Faculty of Electronic and \\ Computer Engineering \\ Universiti Teknikal Malaysia \\ Melaka (UTeM) \\ A.Salleh \\ Faculty of Electronic and \\ Computer Engineering \\ Universiti Teknikal Malaysia \\ Melaka (UTeM)
}

\author{
S. Z. Yahya \\ Faculty of Electronic and \\ Computer Engineering \\ Universiti Teknikal Malaysia \\ Melaka (UTeM) \\ N. R. Mohamad \\ Faculty of Electronic and \\ Computer Engineering \\ Universiti Teknikal Malaysia \\ Melaka (UTeM)
}

\begin{abstract}
The main objective of video compression is to achieve video compression with less possible losses to reduce the transmission bandwidth and storage memory. This paper discusses different approach of video compression for better transmission of video frames for multimedia application. Video compression methods such as frame difference approach, PCA based method, accordion function, fuzzy concept, and EZW and FSBM were analyzed in this paper. Those methods were compared for performance, speed and accuracy and which method produces better visual quality.
\end{abstract}

Keywords: Compression, Losses, Memory, Transmission Bandwidth, Video Compression.

\section{INTRODUCTION}

Moving video images also call video is a main component in internet world today. Video are integrated into the application, website and almost everything that demand the need for moving video images. The need for fast, reliable and high quality video has taken the video compression technology into the next level [1]-[5]. Video compression is a technique that was used reduces redundancy and also reduces the number of bits needed to send or store the video. This paper summarize difference technique or method used in video compression, these method are [6] accordion function, [7] EZW and FMBW, [8] fuzzy concepts, [9] frame difference approach and lastly [10] PCA method.

\section{METHODS OF VIDEO COMPRESSION}

\subsection{Method 1: Accordion Function}

This paper will focus on reducing the storage space for video signal [6]. Figure 1 shows process for block diagram of video compression.

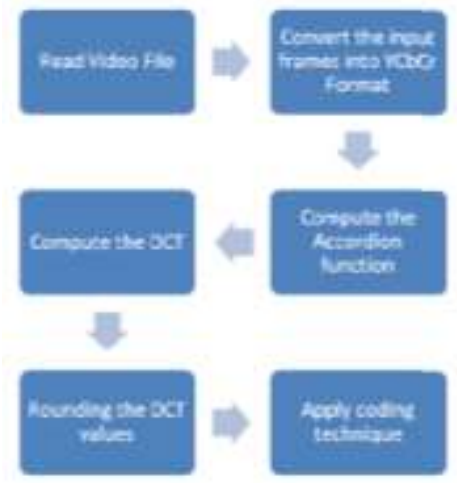

Figure 1: Block diagram of video compression
The technique compressed that involved in this process is removal of spectral redundancy and removal of temporal redundancy. Reduce redundancy which reduce number of bits in order to transmit or keep video data is known as video compression.

\subsubsection{Video compression process}

\subsubsection{Removal of spectral redundancy}

Basic color consists of RED, GREEN and BLUE color, it represent 8 bit of each color which it 24 bits in total to represent any color by adding different proportion of basic color together. This means need more bits to show color information of the given frame and also known as spectral redundancy. The RED, GREEN and BLUE (RGB) need to convert into $\mathrm{YCbCr}$ format in order to reduce number of bits. $\mathrm{Y}$ introduced as Luminance component of the image, $\mathrm{Cb}$ as chrome for blue color and $\mathrm{Cr}$ as chrome for red color. Luminance or Luma represent variation between white to black shades and for chrome it represents color information. By using equation (1) and (3) respectively below the color format of different type of video frames can convert into YCbCr format:

$$
\begin{aligned}
& Y=0.299 R+0.587+0.114 \ldots(1) \\
& \text { For PAL: } \\
& U=0.493(B-Y) \ldots(2) \\
& V=0.877(R-Y) \ldots(3)
\end{aligned}
$$

PAL stands for Phase Alteration in Line. Figure 2 to Figure 3 is result image when the frame is converted into 
$\mathrm{YCbCr}$ format. Figure 3 present the luminance values, Figure 4 present the $\mathrm{Cb}$ values and Figure 5 present the $\mathrm{Cr}$ values for the input frame. From the beginning input video, different frames were taken and assign those into structure and finally were converted into $\mathrm{YCbCr}$ format.

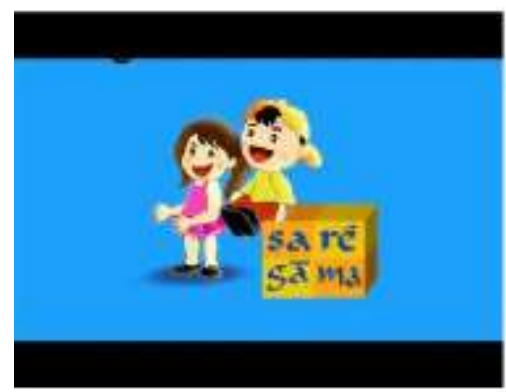

Figure 2: Video Frame

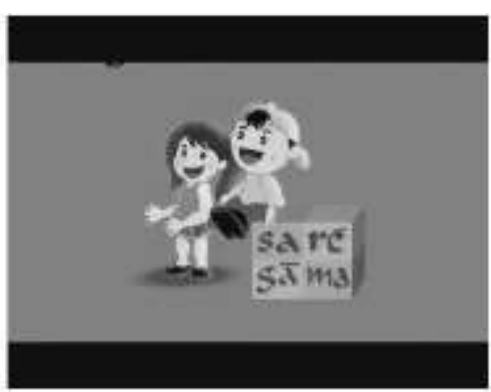

Figure 3: Luminance component

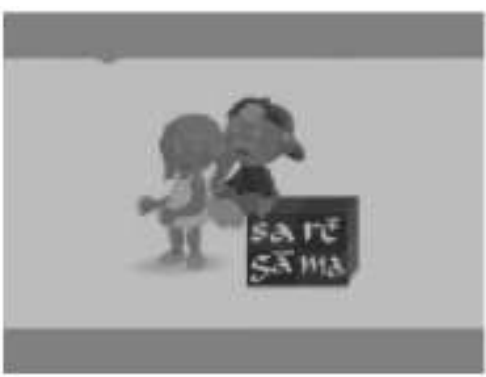

Figure 4: Cb component

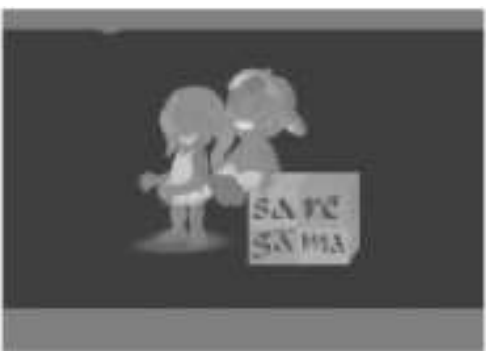

Figure 5: Cr component

Computation of accordion function is presented by four consecutive video frames above as result. These four steps were repeated in Figure 10 for all input video frames.

\subsubsection{Removal of temporal of redundancy}

Temporal redundancy is frequently present between a set of frames which it takes only a few portion of each frame itself involved with any motion that take part.

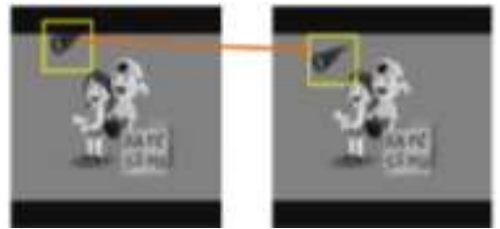

Figure 6: Temporal redundancy

Refer to Figure 6, the yellow box contains motion and all other areas were constant called temporal redundancy. Accordion is a process converted redundancy into spatial redundancy. Temporal redundancy known as non-changing data amount, by placing the successive frames adjacent to each other it can eliminate the constant data in successive frames. It can achieve by using accordion present of 4 successive frames.

Four consecutive groups of frames were taken and merge to create an image in Accordion. The input video of size $\mathrm{M}$ times $\mathrm{N}$ times 500 bits. Figure 7 shows its accordion representation.

The corresponding column pixels were places adjacent to each consecutive frame's column. Refer to input video the consecutive frames from 104 to 108 frame numbers are present in Figure 8 (a), Figure 8 (b), Figure 9 (c) and Figure 9 (d) respectively. Using shift and merge operations, the accordion function was computed on these frames, which represents the temporal redundancy as spatial representation (Figure 10).

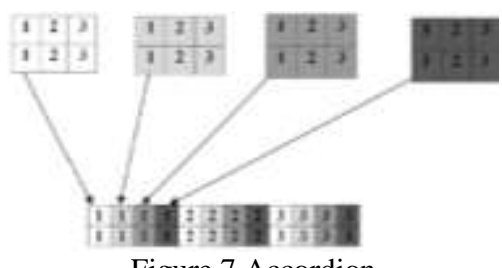

Figure 7 Accordion
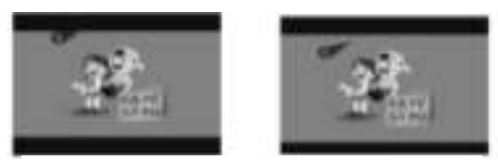

Figure 8 (a) to (b)
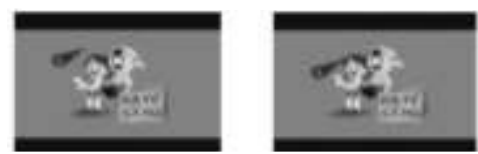

Figure 9 (a) to (b)

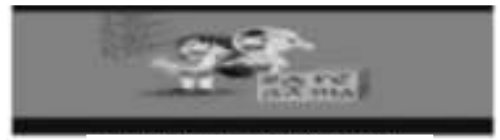

Figure 10

\subsection{Method 2: EZW and FSBM}

Method that been used in this paper is EZW and 7 different algorithms type of block matching algorithms that use in video compression for motion estimation [7]. 7 different algorithms: Exhaustive Search (ES), Three Step Search (TSS), New Three Step Search (NTSS), Simple and Efficient Search (SES), Four Step Search (4SS), Diamond Search (DS) and Adaptive Rood Pattern Search (ARPS). 


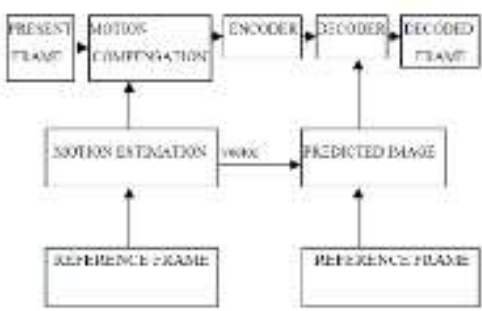

Figure 11: Block diagram for video compression process flow EZW stands for embedded zero tree wavelet algorithm, it simple and effective image compression and make the bits in the bit stream generate in terms of importance yet yielding a complete embedded code. A sequence of binary decisions that remove an image from the null image is representing the embedded code. By using it, at any point encoder locate the encoder can dismiss it, this allow a target rate to be met exactly. Furthermore, EZW consistency produces a compression result to produce a fully embedded bit stream. In order to achieve with this technique it requires no codebooks or training or knowledge of the image store.

\subsubsection{Block Matching Algorithms}

Every pixel within a macro block produce one motion vector for each macro block has some motion activity. The main idea is to divide the current frame into number of macro blocks of fixed size. Also to create a motion vector which it will comprises the location of the macro block of the current frame in the previous frame. As always the macro block is approach as a sequence of sixteen pixels and the search area is seven pixels on all 4 sides in pervious frame of the corresponding macro block. Based on the output of cost functions it can create the matching of one macro block with other.

7 different algorithms type of block matching algorithms:

i. Exhaustive search (ES).

Known as Full Search, is the most expansive algorithm of all. This block matching algorithm calculates the cost function location in the search window as result; it gives the highest Peak-Signal-to-Noise-Ratio (PSNR) and finds the best match that it can get. However there is a disadvantage using ES which is the larger search window the more computations need.

\section{ii. Three Step Search (TSS)}

The earliest in fast block matching back to middle 1980s. TSS search and picks the one that give less cost and can make it as new search origin.

\section{iii. New Three Step Search (NTSS)}

To reduce cost of computational the TSS improved to NTSS, New Three Step Search. NTSS having provisions for half way stop and supply a center biased searching scheme.

\section{iv. Simple and Efficient Search (SES)}

SES is another sub to TSS. In opposite direction it cannot be 2 minimum unimodal surfaces and for eight point which its fixed pattern search of TSS can save on computation by changed it to incorporate. The algorithm same as TSS three steps but the innovation make each steps has more 2 phases.

v. Four Step Search (4SS)
Not to different from NTS, 4SS also have center biased searching itself and halfway stop provision. For the first step, pattern size of 4SS is fixed to $\mathrm{S}=2$ no matter what the $p$ parameter value is.

\section{vi. Diamond Search (DS}

Block matching algorithm DS is exactly the same as 4SS, but the search point pattern which is square changed into diamond pattern and for algorithm there is no limit on the number of steps make this algorithms can find global minimum accurately. 2 difference types of pattern in DS which are Large Diamond Search Pattern (LDSP) and Small Diamond Search Pattern (SDSP). The $1^{\text {st }}$ steps use LDSP and last steps use SDSP.

\section{vii. Adaptive Rood Pattern Search (ARPS)}

ARPS show that the fact of general motion in a frame is coherent. Use the predicted motion vector to check the location pointed and it also can checks at a root distributed points.

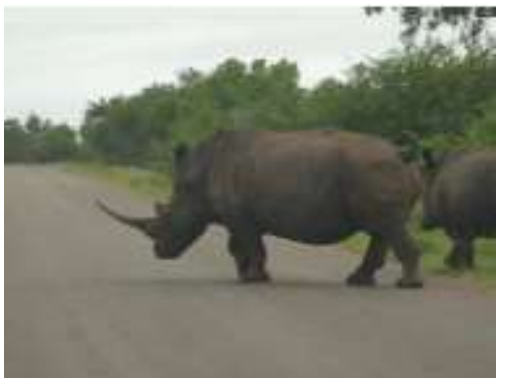

Figure 12: Original image

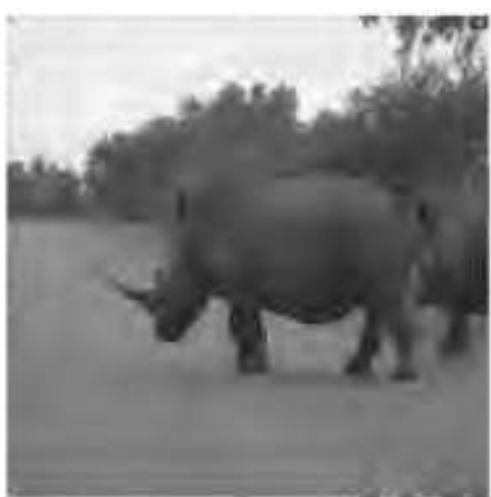

Figure 13: Compressed image using EZW method

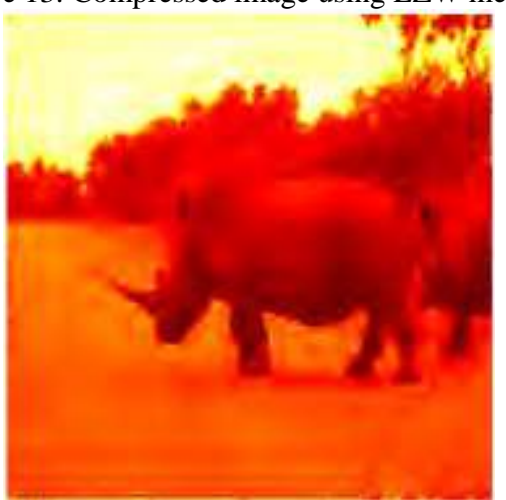

Figure 14: Compression image using artificial colors. 
As result the original size of the video is 25 Mega Byte and the compression video size is reduced to 21.3 Mega Byte. The compression is complete with a good achievement through compression ratio and factor.

\subsection{Method 3: Fuzzy concepts}

This paper focused on the fuzzy concept in the video compression sector with H.264 algorithms and MPEG4 [8]. By applying H.264 in medical video compression and then improve the rate control of H.264 algorithm with better quality. In order to implement it, there is three steps to follow:

i. H.264 need to reviewed and meet the area medical video compression.

ii. A new fuzzy based algorithm is introduce to each frame of the video and make compression of based H.264.

iii. Step is make two comparison between H.264 and MPEG-4 and comparison between JVT-H014 (old version of H.264 algorithm) and proposed fizzy concept. Motion Picture Experts Group (MPEG4) and H.263 are standards that are based on video compression.

a) New fuzzy scheme

The frames will be fuzzified and then fuzzy based segmentation is applied to it is created by a new brand algorithm so each of medical images will have more background that usually it only has ROI. Then the isolation begins. In result it shows the minimum will be compression and the maximum is to the background. The cons with the medical video compression are that some artifacts can always induced inside which make the doctors see a false conclusion. For better result use fuzzy method along with optimum bit rate scheme.

b) Comparison between MPEG-4 and H.264

Table 1 represent the results gain from H.264 and MPEG-4 without put any rate control. For each sequence, as the target bit rates which are 4 bit rates from high to low are selected. The difference between H.264 and MPEG-4, refer to PSNR gain and bit rate saving in the table.

Table 1: Result achieved using MPEG-4 and H.264

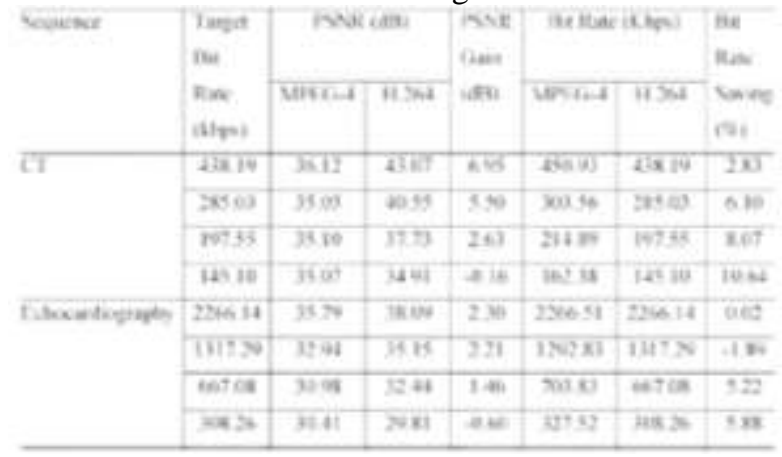

When apply in the test medical video sequence, by referring Table 1 it prove that $\mathrm{H} .264$ performance much better than MPEG-4. Means that H.264 is more effective than MPEG-4 and it is an effective alternative solution for medical video applications.

\subsection{Method 4: Difference frame approach}

This paper discussed about the video compression technique that use algorithm based on frame of video [9]. This technique calculates the frame differencing between the consequences frames according to a specific threshold. This technique concentrated on frames difference approaches that are used in compression technique. There are many stages implemented in this system. Figures 15 show the stages used in this system:

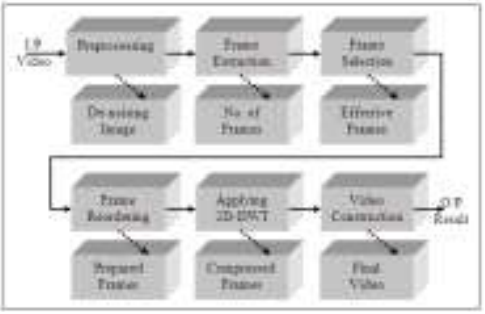

Figure 15: the proposed frame selection key approach

However difference approach is the main core of the system, in which pass the similar frames and select the different frames depends on a certain specified threshold. In addition the selected frames are compressed via applying 2dimensional discrete wavelet transform.

\subsubsection{Frames Difference Approaches for Video Compression}

For removing the lowest frame difference, three different methods are used to remove the identical frames in which frames difference approaches among each consecutive frame of extracted frames are applied. The Summary of the structural diagram of the three approaches is shown in Figure 16.

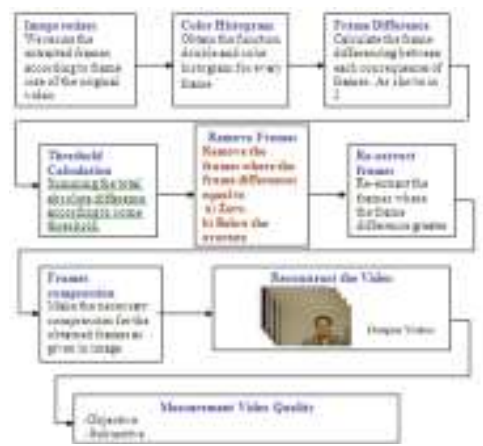

Figure 16: Structural Diagram of frames difference approach

\subsubsection{Zero Difference Approach}

Frames are removed when the gap between any two consecutive frames is zero. This will minimize the number of frames to be re-extracted. Many of the frames difference between consecutive frames are zero; in this case such frames will be removed. 

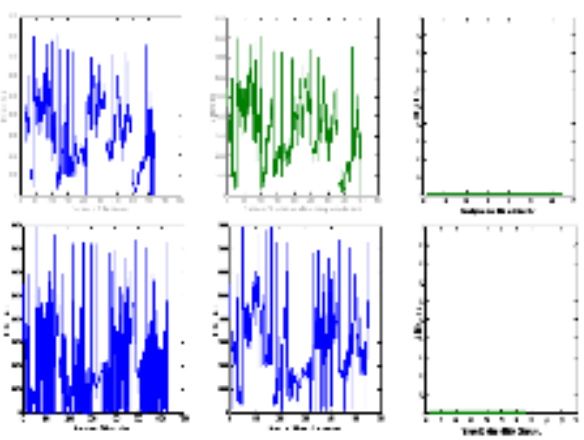

Figure 17: Zero Difference approach

Figures 17, on the left top to bottom represent the frames difference including zero's difference for frames per second 15 and 20 respectively. Middle top to bottom represents frames difference excluding zero's difference frames, while the removed frames in which the frames difference equal to zero are shown in Figures 16 on right top to bottom. As increasing extracting frames per second 10, 15, 20 and 25 , the frames difference equal to zero not necessarily increased, since the frames differences in general decreased as the frames per second increased but not exactly equal to zero.

\subsubsection{Mean Difference Approach}

The mean value of the frames difference is calculated. Mathematically the average is acquired by dividing the sum of the observed values of frames difference by the number of observations. Then remove the frames where the frames difference between any consecutive frames is lower than the mean value of the frames difference.
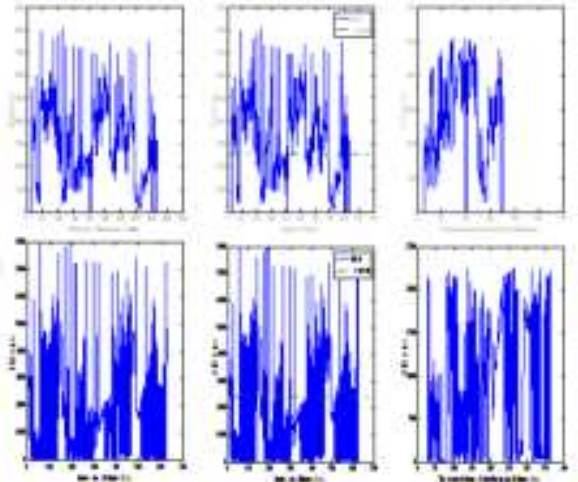

Figure 18: Mean difference approach

Figures 18 from the left top to bottom represent the frames difference including mean's difference for frames per second 15 and 20 respectively. Middle part from top to bottom of the figures represents frames difference including frames below the mean of the overall difference frames; the green line represents the mean of the frames differences. The frames difference above the green line will be maintained and compressed, while the frames difference below the green line will be removed. Left part of the figures represents the frames to be removed. As increasing extracting frames per second, the frames difference decreased and the frames removed are larger

\subsubsection{Percentage Difference Approach}

In this approach different types of videos are examined according to frame details, frame size and the obtained frames differences. This approach concerns mainly in removing the lowest frames difference according to some specific percentage. The percentage depends upon many factors such as compression performance, frame details, frame size and near distance between frames result and analysis.
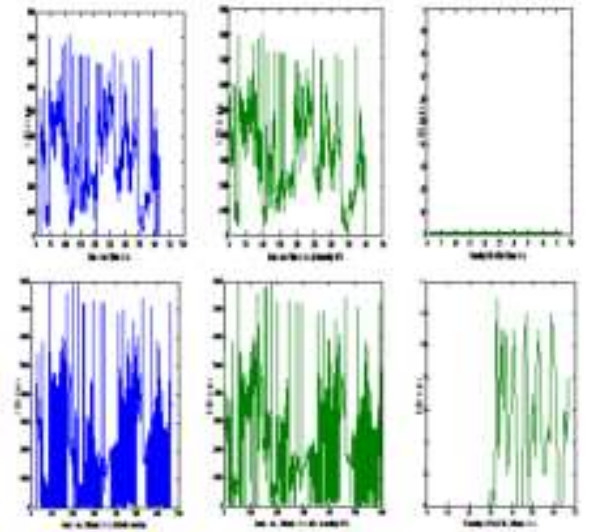

Figure 19: Percentage difference approach

$\mathrm{Fi}$

It the

frames difference for different frames per second $15, \& 20$ respectively. Middle part from top to bottom of the Figures 4.3 represents frames difference after removing $10 \%$ of the lowest frames difference. Left Part of the figures 4.3 represents the frames to be removed. Also different percentages like $15 \%, 20 \%$ were removed of the frames difference.

\subsection{Method 5: PCA method}

This paper brings up the use of a statistical approach for video compression as new Principle Component Analysis (PCA) based method [10]. Based on necessary of accuracy this method reveals the features of video frames and process them flexibly. This main idea improves the quality of compression effectively. Furthermore this method also focused on the truth that video is a composition of correlated and sequential frames or image, so that the PCA can be applied in and the advantages of using this method is it does not reduce the bandwidth of frequency response, so the progress of frames won't disappear.

\subsubsection{PCA method}

The values of the components of the matrixes are Mgrayscale $\mathrm{N} \times$ Pimages. $\mathrm{N} \times$ Pgrayscale images are equivalent to $\mathrm{N} \times \mathrm{P}$ matrixes. By applying this method, images are move to another part. All images are put in $\boldsymbol{X}$ matrix that its elements are the intensity values of images.

Considering the first $\mathrm{k}$ Eigen vectors from the $\mathrm{M}$ Eigen vectors $(K<M)$, the $\mathbf{X}$ matrix that is a little bit different from $\mathbf{X}$ matrix. The $\mathbf{X}$ matrix is compressed matrix which it gain from $\mathbf{X}$. So the compression ratio - the ratio of the required memory to save $\mathbf{X}$ to the neccasary memory to keep $\mathbf{X}$ - can be determined as below:

$$
\text { Memory ratio }=\frac{\text { requiredmemorytasave } x}{\text { requiredmemorytosave } x}
$$

\subsubsection{IMPROVED PCA}

It is available to develop the quality of image exactly and knew the most inaccurate pixels in the reassemble frame or images. The $\mathbf{Y} \mathrm{k}$ matrix is changed to $\hat{\mathrm{Y}} \mathrm{k}$, which to compensate the effect of error the nonzero columns in the bottom of the matrix are applied: 


$$
\widehat{\mathbf{Y}}_{k}=\left[\begin{array}{cccccc}
\mathrm{Y}(1,1) & \ldots & \mathrm{Y}(1, \alpha) & \ldots & \mathrm{Y}\left(1, \mathrm{~N}^{2}-1\right) & \mathrm{Y}\left(1, \mathrm{~N}^{2}\right) \\
\mathrm{Y}(2,1) & \ldots & \mathrm{Y}(2, \alpha) & \ldots & \mathrm{Y}\left(2, \mathrm{~N}^{2}-1\right) & \mathrm{Y}\left(2, \mathrm{~N}^{2}\right) \\
\vdots & \vdots & \vdots & \vdots & \vdots & \vdots \\
\mathrm{Y}(\mathrm{k}, 1) & \ldots & \mathrm{Y}(\mathrm{k}, \alpha) & \ldots & \mathrm{Y}\left(\mathrm{k}, \mathrm{N}^{2}-1\right) & \mathrm{Y}\left(\mathrm{k}, \mathrm{N}^{2}\right) \\
0 & 0 & \mathrm{Y}(\mathrm{k}+1, \alpha) & \ldots & 0 & 0 \\
0 & 0 & \mathrm{Y}(\mathrm{k}+2, \alpha) & \ldots & 0 & 0 \\
\vdots & \vdots & \vdots & \vdots & 0 & 0 \\
0 & 0 & \mathrm{Y}(\mathrm{M}, \alpha) & \ldots & 0 & 0
\end{array}\right]_{\mathrm{MxNN}^{2}}
$$

\subsubsection{DPCA Method}

To get image vectors of images construct a high dimensional image vector space the image matrixes need to changed to vectors. This method uses $2 \mathrm{D}$ matrixes rather than 1D vectors in conventional PCA and 2DPCA has two fundamental benefit over conventional PCA

a) Easy to design accurate covariance matrixes

b) Because of covariance matrixes size are smaller it took less time to determine corresponding eigenvectors.

\subsubsection{Improved PCA and 2DPCA for Video}

This part represent the results of applying three mentioned methods above: PCA, Improved PCA and 2DPCA for compression of fourteen sample frames are show. The frames are pick from throwing an apple in a white background. Figure 19 shows the original frames. The frames are organize from the top down and left to right.

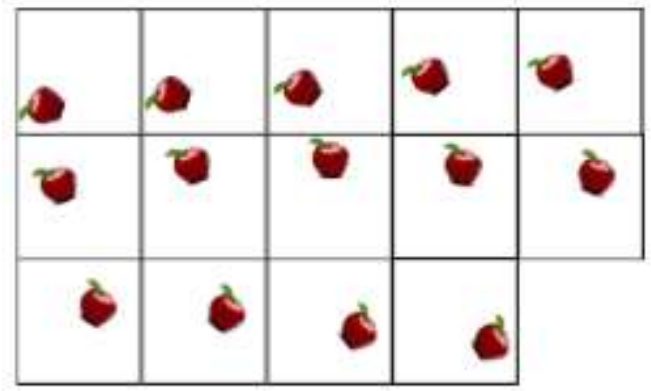

Figure 20: Based on the background of PCA, 2DPCA and improved PCA in face database compression, the improved PCA shows better results than two other methods.

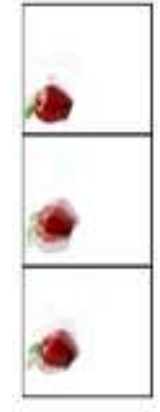

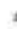

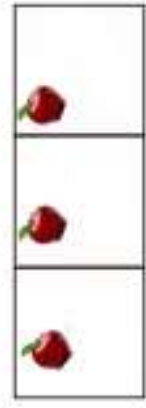

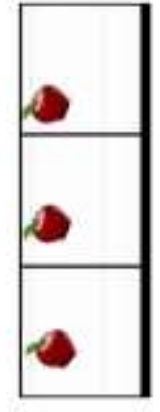

Figure 21: PCA method added fade effects

Figure 21 shows the first three frames of applying mentioned triple methods with same bitrates to the original frames. As it seen, the PCA method added fade effects to the reconstructed frames and applying 2DPCA causes vertical lines and black bands to the frames. So in this application (video compression), the best performance is provided by improved PCA method.
3. DISCUSSIONS

\begin{tabular}{|l|l|}
\hline Method & \multicolumn{1}{|c|}{ Summary } \\
\hline \multirow{5}{*}{ Accordion function } & $\begin{array}{l}\text { In this proposed technique, } \\
\text { The compressions remove } \\
\text { the spectral redundancy and } \\
\text { temporal redundancy by } \\
\text { using Discrete Cosine } \\
\text { Transform and convert into } \\
\text { spatial redundancy. }\end{array}$ \\
\hline EZW is used as intra \\
compression and seven \\
different block matching \\
algorithm are used for \\
motion estimation in video \\
compression the result are \\
much better if using SPIHT \\
algorithm.
\end{tabular}

\section{CONCLUSIONS}

In this paper, five difference methods for video compression are reviewed and discussed. There are still a lot of possibilities for the improvement for lossless, small size, and high quality video for the mentioned method, although these method have a pros and cons. In the end, the best method for video compression are the one that can bring faster, reliable and smaller in size without affecting much on the video quality. All of these requirements is necessary for multimedia application, for user to enjoy the video.

\section{ACKNOWLEDGMENTS}

We are grateful to Centre for Telecommunication Research and Innovation (CeTRI) and Universiti Teknikal Malaysia Melaka (UTeM) through PJP/2013/FKEKK (29C)/S01215 for their kind and help for supporting financially and supplying the electronic components and giving their laboratory facility to complete this study.

\section{REFERENCES}

[1] N. M. Z. Hashim, A. F. Jaafar, Z. Zakaria, A. Salleh, and R. A. Hamzah, "Smart Casing for Desktop Personal Computer," International Journal of Engineering and Computer Science (IJECS), vol. 2, no. 8, pp. 2337 $2342,2013$. 
[2] N. M. Z. Hashim, N. H. Mohamad, Z. Zakaria, H. Bakri, and F. Sakaguchi, "Development of Tomato Inspection and Grading System using Image Processing," International Journal Of Engineering And Computer Science (IJECS), vol. 2 no. 8, pp. 2319-2326, 2013.

[3] N. M. Z. Hashim, N. A. Ali, A. Salleh, A. S. Ja'afar, and N. A. Z. Abidin, "Development of Optimal Photosensors Based Heart Pulse Detector," International Journal Of Engineering and Technology (IJET), vol. 5, no. 4, pp. 3601-3607, 2013.

[4] N. M. Z. Hashim, N. M. T. N. Ibrahim, Z. Zakaria, F. Syahrial, and H. Bakri, "Development New Press Machine using Programmable Logic Controller," International Journal of Engineering and Computer Science (IJECS), vol. 2, no. 8, pp. 2310-2314, 2013.

[5] N. M. Z. Hashim, N. A. Ibrahim, N. M. Saad, F. Sakaguchi, and Z. Zakaria, "Barcode Recognition System," International Journal of Emerging Trends \& Technology in Computer Science (IJETTCS), vol. 2, no. 4, pp. 278-283, 2013.

[6] Mitesh Shah, Hetal Patel, "Design of a New Video Compression Algorithm Using Accordion Function", International Journal of Science and Modern
Engineering (IJISME) ISSN: 2319-6386, Volume-1, Issue-6, May 2013.

[7] Sangeeta Mishra, Sudhir Savarkar, "Video Compression Using EZW and FSBM", International Journal of Scientific and Research Publications, Volume 2, Issue 10, October 2012, ISSN 2250-3153.

[8] MuzhirShaban Al-Ani and Talal Ali Hammouri, Video Compression Algorithm Based on Frame Difference Approaches International Journal on Soft Computing (IJSC) Vol.2, No.4, November 2011.

[9] J. Mohanalin Rajarathnam, A novel Fuzzy based Medical video compression using H.264, Georgian Electronic ScientificJournal: Computer Science and Telecommunications 2008|No.3 (17).

[10] Mostafa Mofarreh-Bonab, Mohamad Mofarreh-Bonab, Adaptive Video Compression using PCA Method International Journal of Computer Applications (0975 8887) Volume 44- No.21, April 2012. 